\title{
Concepção de profissionais de saúde sobre o papel das unidades básicas nas redes de atenção do SUS/Brasil
}

\author{
The viewpoint of health professionals on the role of basic units \\ in the healthcare network of the Brazilian Unified Health System
}

Paulo de Tarso Puccini ${ }^{1}$

Vitória Keddy Cornetta ${ }^{2}$

Tânia Zogbi Sahyom ${ }^{3}$

Isabel Cristina Pagliarini Fuentes ${ }^{4}$

Luiza Maria Gimenez Botta ${ }^{5}$

Rosana Fiorini Puccini ${ }^{6}$

${ }^{1}$ Universidade de São Paulo. Av. Piassanguaba, 1923, Planalto Paulista. 04060-003 São Paulo SP. paulopuccini@gmail.com ${ }^{2}$ Faculdade de Saúde Pública, Universidade de São Paulo.

${ }^{3}$ Secretaria Municipal de Saúde, Prefeitura de EmbuGuaçu.

${ }^{4}$ Secretaria Municipal de Saúde, Prefeitura de Embu das Artes.

${ }^{5}$ Hospital Municipal Vereador José Storopolli São Paulo (2006-2008).

${ }^{6}$ Disciplina de Pediatria Geral e Comunitária,

Departamento de Pediatria, Escola Paulista de

Medicina, Universidade

Federal de São Paulo

(UNIFESP).

\begin{abstract}
In the process of creation of the Unified Health System (SUS) as a universal policy seeking to ensure comprehensive care, unscheduled assistance in primary healthcare units (UBS) is an unresolved challenge. The scope of this paper is to analyze the viewpoint of health professionals on the role of primary healthcare units in meeting this demand. It is a transversal study of qualitative data obtained through questionnaires and interviews with 106 medical practitioners from 6 emergency medical services and 190 professionals from 30 units. They explained why people seek emergency care for occurrences pertaining to primary care. The content analysis technique with thematic categories was used for data analysis. Lack of resources and problems with primary health unit work processes (50.8\%) were the reasons most frequently cited by emergency care physicians to explain this inadequate demand. Only $33.3 \%$ of the health unit professionals agreed that these occurrences should be attended in the primary healthcare services. The limited viewpoint of the role of health services on the unscheduled care, particularly among primary care professionals, possibly leads to restrictive practices for access by the population.
\end{abstract}

Key words Primary healthcare services, Comprehensive healthcare, Access to health services
Resumo No processo de construção do SUS, como politica universal e que busca assegurar a integralidade das ações, a assistência à demanda não agendada na atenção básica constitui desafio não superado. Objetivo: analisar a concepção de profissionais de saúde quanto ao papel das unidades básicas de saúde (UBS) na assistência a essa demanda. Método: estudo transversal considerou dados qualitativos obtidos por meio de questionários e entrevistas. Incluídos 106 médicos de 6 PS/AMA e 190 profissionais de 30 UBS. Estes opinaram sobre razões de a população procurar PS/ AMA por ocorrências nosológicas próprias da atenção básica. Para análise, utilizou-se a técnica de análise do conteúdo, segundo categorias temáticas. Resultados: insuficiência de recursos e dificuldades no processo de trabalho das UBS $(50,8 \%)$ foi a explicação mais frequentemente apontada por médicos de PS/AMA para justificar procura inadequada. Apenas 33,3\% dos profissionais das UBS opinaram que essas ocorrências deveriam ser atendidas na atenção básica. Conclusões: A concepção limitada sobre o papel das UBS na atenção a essa demanda, sobretudo de profissionais da atenção básica, resulta, possivelmente, em práticas restritivas ao acesso da população.

Palavras-chave Serviços básicos de saúde, Assistência integral à saúde, Acesso aos Serviços de Saúde 


\section{Introdução}

No processo de construção do SUS como política universal e que busca assegurar a integralidade das ações, o equacionamento da atenção à demanda não agendada na rede básica é essencial e constitui um desafio ainda não superado. A não-resposta a essa demanda, a rigidez dos programas e a não-incorporação de forma ágil de novas necessidades em saúde resultam em desnecessária procura da população por unidades de urgência-emergência ou de ambulatórios de especialidades e, em consequência, as unidades básicas perdem sua condição de local onde se privilegiam o vínculo, a coordenação e continuidade dos cuidados e a credibilidade para realizar ações de promoção e prevenção $0^{1-4}$.

Estudos realizados em serviços de emergência que procuram compreender aspectos relacionados ao perfil de morbidade e motivações dessa procura tem apontado sistematicamente que parcela significativa das ocorrências poderia ser resolvida na atenção básica ${ }^{5-7}$.

O questionamento sobre possíveis limitações dos serviços de atenção básica na assistência à demanda não agendada e preocupações de que essa assistência poderia levar a um fortalecimento de ações historicamente distorcidas, caracterizadas por atendimentos pontuais, sem continuidade ou vínculo com os serviços de saúde, tipo queixa-conduta, tem dificultado a estruturação de um modelo que dê conta dessa questão ${ }^{8}$. Ocorre que atender prontamente, e com médicos, é objetivamente fundamental para a atenção básica. A questão que se coloca é como fazer isso de forma racional, ampla, estável e articulada com os princípios gerais de defesa do direto de todos à saúde; como fazer isso de forma a ampliar a assistência imediata, garantindo sua continuidade, incluindo a contribuição de diferentes profissionais de saúde que permita uma ampliação do trabalho interdisciplinar e o cuidado integral e continuado $^{9,10}$. O processo de conversão da rede básica em rede de saúde da família e a modalidade de serviço de saúde intermediária entre as unidades básicas e os prontos-socorros (Ambulatório de Assistência Médica Ambulatorial - AMA, no município de São Paulo, e a Unidade de Pronto Atendimento - UPA, na sua versão inicial, implementada pelo Ministério da Saúde) podem resultar em limitado papel da atenção básica na rede de serviços com possíveis repercussões para a saúde da população, sobretudo diante de condições que, em especial, exigem integralidade e continuidade dos cuidados ${ }^{11}$.
Constitutivas das dificuldades objetivas que a atenção básica tem apresentado em responder a essa demanda, verificam-se orientações à população e concepções controversas em relação ao seu papel na assistência à demanda não agendada, tanto de profissionais de serviços de urgência e emergência como daqueles que atuam nas unidades básicas de saúde. Uma compreensão clara e abrangente sobre o papel da atenção básica à saúde é fundamental para os profissionais que atuam em diferentes tipos de serviços, sobretudo nas unidades básicas e nos serviços de urgência e emergência, e também para os gestores, visando ao desenvolvimento da educação permanente e reorientação nos processos de trabalho, para que essas ações sejam mais efetivas e articuladas.

Este estudo foi realizado com o objetivo de analisar a concepção de médicos de serviços de urgência e emergência e de profissionais da atenção básica no que se refere ao papel das unidades básicas numa rede de atenção à saúde e, dessa forma, contribuir para a compreensão e o desenvolvimento da articulação entre os diferentes níveis e pontos de atenção, visando à consolidação da integralidade da assistência e a continuidade dos cuidados, essenciais no processo de construção do SUS.

\section{Método}

Este estudo é parte do Projeto MABS - Análise de um indicador de monitoramento dos serviços de atenção básica de saúde em diferentes modelos assistenciais - o qual toma como base o conceito de eventos sentinela e, assim, partindo da hipótese de que determinadas ocorrências nosológicas atendidas em pronto-socorro (PS) ou Ambulatório Médico Assistencial (AMA) podem estar relacionadas a falhas da atenção básica, analisa a utilização dessas ocorrências na formulação de um indicador para o monitoramento da atenção básica à saúde na reorientação do seu papel integral ${ }^{12}$.

Este projeto foi desenvolvido em municípios da Região Metropolitana de São Paulo - Embu, Embu-Guaçu e São Paulo (Supervisão Técnica de Saúde da Vila Maria), visando a incluir distintas realidades e opções de organização da atenção básica, que permitissem conhecer e explorar de forma abrangente as relações entre ocorrências previamente escolhidas e atendidas em PS/ AMA e falhas da atenção básica. A população $0^{13} \mathrm{e}$ a respectiva rede de serviço de cada município, no ano de 2007, são descritas a seguir: Embu - 
250.532 habitantes, 12 unidades básicas de saúde (6 com modelo tradicional e 6 com Programa de Saúde da Família) e 2 PS; Embu-Guaçu - 76.639 habitantes, 6 unidades básicas de saúde (tradicional/PSF simultaneamente) e 2 PS; Supervisão Técnica de Vila Maria (Distritos de Vila Maria, Vila Guilherme e Vila Medeiros) do município de São Paulo - 284.337 habitantes, 12 unidades básicas ( 10 com modelo tradicional e 2 com Programa de Saúde da Família), 1 PS de hospital geral (Hospital Municipal Vereador José Storopolli) e 1 Ambulatório Médico Assistencial (AMA). No total foram incluídos: 5 PS, 1 AMA e 30 unidades básicas de saúde (UBS).

\section{Tipo de estudo}

O Projeto MABS foi conduzido como estudo transversal que incluiu gestores e gerentes dos serviços de saúde como pesquisadores participantes, os quais foram envolvidos em todas as etapas de sua realização. A coleta de dados quantitativos e qualitativos ocorreu em três momentos sucessivos no período de 05/2006 a 02/2008. A formulação do indicador de monitoramento da atenção básica (I_mabs) proposto no estudo resultou de uma composição que incluiu: frequência das hipóteses diagnósticas escolhidas por serem consideradas como mais apropriadas para atendimento na atenção básica (HDE); escore_UBS constituído por avaliação qualiquantitativa das UBS, pontuada com base em sua atuação referente a diferentes aspectos organizacionais, especialmente a assistência à demanda não agendada; e, análise crítica do indicador utilizando-se dados qualitativos. Nesta análise, empregando-se a triangulação de métodos, foram discutidos os valores quantitativos obtidos com o indicador (I_mabs), suas diferenças entre as áreas/UBS de procedência das pessoas atendidas nas unidades de urgência e emergência, confrontando-o com os discursos obtidos por meio de entrevista com os usuários atendidos e por meio de questionários semiestruturados aplicados aos médicos dos serviços de urgência e emergência e aos profissionais (médicos, enfermeiros, auxiliares e técnicos de enfermagem) das unidades básicas de saúde. A delimitação do conjunto de ocorrências nosológicas, possivelmente associadas às falhas da atenção básica, tal como propõe o conceito de evento sentinela, considerou a opinião de médicos de serviços de urgência e emergência de seis PS/AMA que atuavam no período diurno dos três municípios. As ocorrências nosológicas indicadas pelos médicos desses serviços (Quadro 1) foram utilizadas, posteriormente, na formulação da pergunta dirigida aos profissionais da atenção básica sobre o papel da unidade básica no atendimento a essas afecções.

Respondendo às perguntas abertas dos questionários, os médicos dos serviços de urgência e emergência e os profissionais da atenção básica apontaram possíveis razões que determinariam essa procura inadequada. De 121 médicos existentes nos seis PS/AMA dos três municípios, responderam ao questionário $106(87,6 \%)$. Na atenção básica, responderam ao questionário uma amostra constituída por 190 profissionais que atuavam nas 30 unidades básicas integrantes das três redes de saúde participantes do estudo. Essa amostra, não aleatória, incluiu um a dois profissionais de saúde, de cada uma das seguintes categorias: médicos (generalistas, clínicos, ginecoobstetras ou pediatras), enfermeiros, auxiliares e técnicos de enfermagem. Em relação aos auxiliares e técnicos de enfermagem o questionário foi dirigido àqueles que atuavam no acolhimento (Tabela 1).

Neste artigo são abordados os resultados obtidos por meio dos questionários aplicados aos médicos dos serviços de urgência e emergência e aos profissionais de saúde das unidades básicas de saúde. Para a análise dos depoimentos utilizou-se a técnica de análise do conteúdo segundo categorias temáticas, a qual realiza operações de desmembramento do texto em unidades de significado segundo categorias pré-estabelecidas. Segundo Bardin ${ }^{14}$, embora limitada, essa é uma técnica rápida, eficaz que pode ser aplicada a discursos diretos e simples.

O projeto foi aprovado pelas secretarias municipais de saúde dos três municípios e pelo Comitê de Ética e Pesquisa da Faculdade de Saúde Pública da USP. Todos os profissionais e usuários participantes do estudo assinaram termo de consentimento livre e esclarecido.

\section{Resultados}

Os resultados obtidos a partir dos questionários aplicados aos profissionais são apresentados segundo local de atuação: A) médicos que atuam em PS/AMA; e, B) profissionais (médicos, enfermeiros, auxiliares e técnicos de enfermagem) que atuam em unidades básicas de saúde. 
Quadro 1. Ocorrências atendidas em unidades de urgência e emergência escolhidas para o monitoramento da atenção básica. Projeto MABS - Região Metropolitana de São Paulo, 2006-2008.

\begin{tabular}{|c|c|c|}
\hline Grupo & CID_10 & Ocorrência \\
\hline IVAS & $\begin{array}{l}\mathrm{J} 03 \\
\mathrm{~J} 00 \text { e J11 } \\
\text { H65 } \\
\text { J01 }\end{array}$ & $\begin{array}{l}\text { Amigdalite } \\
\text { Nasofaringite } \\
\text { Otites } \\
\text { Sinusite }\end{array}$ \\
\hline Respiratórias & $\begin{array}{l}J 45 \\
\text { R05 } \\
\text { R06 }\end{array}$ & $\begin{array}{l}\text { Asma brônquica } \\
\text { Tosse } \\
\text { Dispneia, anormalidade da respiração }\end{array}$ \\
\hline Gastrointestinais & $\begin{array}{l}\text { A09 } \\
\mathrm{k} 30\end{array}$ & $\begin{array}{l}\text { Diarreia e gastroenterite, infecciosa presumível } \\
\text { Dispepsia }\end{array}$ \\
\hline Dorsalgias & M54 & Dorsalgia, cervicalgia, dor lombar baixa \\
\hline Urinárias & N39 & $\begin{array}{l}\text { Outros transtornos do trato urinário (ITU), N30 Cistite, } \\
\text { N34 Uretrite, síndrome uretral }\end{array}$ \\
\hline HAS e Diabetes & $\begin{array}{l}\text { I10 } \\
\text { E14 }\end{array}$ & $\begin{array}{l}\text { HAS } \\
\text { Diabetes }\end{array}$ \\
\hline Saúde bucal & K08 & Outros transtornos dos dentes, dor de dente \\
\hline Orientação técnica & $\begin{array}{l}\mathrm{Z} 00 \\
\mathrm{Z} 01 \\
\mathrm{Z} 02 \\
\mathrm{Z} 76\end{array}$ & $\begin{array}{l}\text { Consulta geral sem queixa } \\
\text { Exames (pedidos ou dúvidas) } \\
\text { Consulta com finalidades administrativas (atestado, } \\
\text { especialista) } \\
\text { Medicação }\end{array}$ \\
\hline
\end{tabular}

Tabela 1. Profissionais entrevistados, segundo categoria profissional, tipo de serviço e rede de saúde participante. Projeto MABS - Região Metropolitana de São Paulo, 2006-2008.

\begin{tabular}{|c|c|c|c|c|c|c|}
\hline \multirow[b]{2}{*}{ Local: rede de saúde } & \multirow[b]{2}{*}{ Tipo de serviço } & \multicolumn{3}{|c|}{ Categoria profissional } & \multirow[b]{2}{*}{ Total } & \multirow[b]{2}{*}{$\%$} \\
\hline & & Médico & Enfermeiro & Enfermagem $^{*}$ & & \\
\hline \multirow[t]{4}{*}{ Embu } & PS Annete & 8 & 0 & 0 & 8 & 2,7 \\
\hline & PS Central & 14 & 0 & 0 & 14 & 4,7 \\
\hline & 12 Unidades Básicas & 42 & 11 & 32 & 85 & 28,7 \\
\hline & Total & 64 & 11 & 32 & 107 & 36,1 \\
\hline \multirow[t]{4}{*}{ Embu-Guaçu } & Un. Mista de Saúde & 11 & 0 & 0 & 11 & 3,7 \\
\hline & PS Cipó & 6 & 0 & 0 & 6 & 2,0 \\
\hline & 6 Unidades Básicas & 19 & 4 & 13 & 36 & 12,2 \\
\hline & Total & 36 & 4 & 13 & 53 & 17,9 \\
\hline \multirow[t]{4}{*}{ São Paulo - V. Maria } & AMA-VG & 17 & 0 & 0 & 17 & 5,7 \\
\hline & PS do HVM & 50 & 0 & 0 & 50 & 16,9 \\
\hline & 12 Unidades Básicas & 68 & 1 & 0 & 69 & 23,3 \\
\hline & Total & 135 & 1 & 0 & 136 & 45,9 \\
\hline Total & & 235 & 16 & 45 & 296 & 100,0 \\
\hline
\end{tabular}

*Enfermagem $=$ auxiliar e técnico de enfermagem 


\section{A) Médicos que atuam em PS/AMA}

Estes profissionais, após indicarem ocorrências nosológicas atendidas nos PS/AMA que, na opinião deles, deveriam ser atendidas nas unidades básicas, conforme apresentado e sintetizado no Quadro 1 opinaram sobre o que determinaria essa procura inadequada ( $\mathrm{Na}$ sua opinião, o que determinaria essa procura inadequada?). Em relação a esta pergunta, foram identificadas cinco categorias temáticas, descritas a seguir, e a frequência das mesmas encontra-se na Tabela 2.

- Insuficiência e inadequação de recursos na UBS. Nesta categoria temática, são organizadas as opiniões que apontam como razões dessa procura inadequada: falta de unidades, de médicos, de qualificação técnica do profissional e de acesso ágil aos exames; insuficiência das ações de promoção e prevenção já desenvolvidas, que, segundo esses profissionais, deveriam ser intensificadas. Não há uma crítica ao processo de trabalho nas UBS e suas formas de organização, no que se refere à assistência à demanda não agendada.

Precisa contratar mais profissionais da área, assim diminui espaço de tempo de retorno nos postos de saúde. Em alguns casos, os retornos são marcados em média de 6 a 9 meses.

Faltam recursos materiais e de pessoal na UBS. Falta de investimento na medicina preventiva através dos PSF e melhorar a qualificação do médico do Posto de saúde.

É muito demorado o tempo de agendamento das consultas nas UBS. É preciso ampliar os horários de atendimento nas UBS e melhorar a agilidade dos serviços de diagnóstico.
O número de UBS é insuficiente e para aumentar número atendimentos é necessário contatar mais profissionais e com salários mais atrativos.

Prestar um serviço eficiente de prevenção, investigação e tratamentos adequados com o propósito de oferecer uma boa qualidade de vida.

Aumentar o número de funcionários para acolhimento domiciliar. Otimizar o PSF, não fazendo o PSF funcionar como um grande PA. Capacitação dos profissionais da área da saúde. Treinamentos participativos.

- Incapacidade do tipo de organização das unidades básicas. Nesta categoria são apresentadas as opiniões que apontam para a incapacidade das unidades básicas em responderem à demanda não agendada, necessitando de, além de mais recursos, maior acesso aos cuidados com mudanças no processo de trabalho: maior espaço na agenda, rodízio de profissionais, atender diante do problema e não somente quando há consulta marcada.

A maioria dos pacientes vem ao PS afirmando não terem sido atendidos no posto de saúde por não terem encaminhamento e pela demora no atendimento.

O paciente deveria ser atendido (ter acolhimento imediato) também diante de seu quadro de saúde e não só quando tem consulta marcada.

A UBS deveria garantir atendimento do paciente na procura sem agenda.

Deveria ser estabelecido aos postos a obrigatoriedade dos atendimentos e pronto-atendimento de doenças.

$\mathrm{Na}$ ausência de encaixes por parte da UBS o paciente procura os PS.

Tabela 2. Categorias discursivas, segundo profissionais entrevistados em prontos-socorros e AMA e localização da unidade. Projeto MABS - Região Metropolitana de São Paulo, 2006-2008.

\begin{tabular}{|c|c|c|c|c|c|c|c|c|}
\hline \multirow[b]{2}{*}{ Categoria temática ${ }^{*}$} & \multicolumn{2}{|c|}{ Embu } & \multicolumn{2}{|c|}{ Embu-Guaçu } & \multicolumn{2}{|c|}{ São Paulo } & \multicolumn{2}{|c|}{ Total } \\
\hline & $\mathbf{N}$ & $\%$ & $\mathbf{N}$ & $\%$ & $\mathrm{~N}$ & $\%$ & $\mathrm{~N}$ & $\%$ \\
\hline 1 & 13 & 56,0 & 9 & 43,8 & 33 & 50,3 & 55 & 50,8 \\
\hline 2 & 1 & 3,8 & 2 & 12,4 & 4 & 6,2 & 7 & 6,1 \\
\hline 3 & 2 & 11,2 & 5 & 33,3 & 10 & 14,3 & 17 & 14,5 \\
\hline 4 & 2 & 9,9 & 1 & 10,5 & 10 & 13,7 & 13 & 13,2 \\
\hline 5 & 3 & 14,4 & 0 & - & 6 & 9,2 & 9 & 9,6 \\
\hline Total & 21 & 100,0 & 17 & 100,0 & 63 & 100,0 & 101 & 100,0 \\
\hline
\end{tabular}

Sem resposta $=5$

1. Insuficiência e inadequação de recursos na UBS.

2. Incapacidade do tipo de organização das unidades básicas.

3. Falta de informação e "educação da população" na utilização adequada dos serviços de saúde.

4. Poder atrativo do PS em relação à oferta ágil de consulta e exames.

5. Incapacidade do poder público gerir a saúde. 
. Falta de informação e "educação da população" na utilização adequada dos serviços de saúde. Nesta categoria são incluídas as opiniões dos profissionais que consideram necessário "educar a população" para que ela possa superar um comportamento negligente com a saúde, de nãoseguimento das orientações, o que resultaria numa valorização exagerada da procura por atendimento imediato. É necessário "educar a população" visando a estimular o autocuidado, evitando assim o adoecer, além de orientar como utilizar os serviços de saúde nas diferentes situações.

Falta divulgação na imprensa sobre qual situação deve-se procurar o PS ou a UBS, é preciso divulgar, divulgar, divulgar.

Falta de orientação a nossa população. Informações sobre que patologias podem e devem ser acompanhadas nas UBS e quais as que devem ser atendidas em hospitais. É preciso divulgação permanente em hospitais e UBS destes diferentes niveis de atenção à saúde. Campanhas com estagiários de áreas primárias à saúde para informação da população.

Palestras de orientação de doenças na sala de espera. Vídeos, medicina de família.

Aumentar o número de funcionários para visita domiciliar, para que o usuário saiba se há necessidade de procurar UBS, PS ou unidade mista. Fazer grupos nas UBS para orientá-los sobre: o que é PS? O que é unidade básica, posto de saúde ou centro de especialidade?

São necessárias mais campanhas educativas à população, em mídia de grande penetração, para entender os papéis dos serviços.

Falta de informação da população que é imediatista e incentivada a procurar o PS por causa do fornecimento indiscriminado de atestados. Sensacionalismo da imprensa.

. Poder atrativo do PS em relação à oferta ágil de consulta e exames. Diante desse poder atrativo desse tipo de serviço, nesta categoria incluemse as opiniões sobre a necessidade de adotar medidas restritivas de acesso ao atendimento no PS, sugerindo a implantação de triagem ou o atendimento de demanda referenciada (porta fechada).

A procura exagerada pelos PS pode ser resolvida com uma triagem no pronto-socorro, descartando os casos que não sejam urgência e emergência.

Os PS poderiam estabelecer triagem por médico urgencista, residentes ou médico da equipe fazendo rodízio.

Haver uma triagem (médica) no pronto-atendimento para evitar abrir fichas desnecessárias que tomam o tempo do médico e prejudicam o atendimento dos pacientes com urgência.
Como há falta de vontade do paciente de "esperar" o dia da consulta do posto de saúde é preciso melhorar a orientação ou até mesmo criar uma triagem antes do paciente fazer a ficha para atendimento.

Falta de cartão para saúde (como se fosse cartão do convênio) para fazer a computação integrada (integrar os postos e PS). Obs: várias vezes, por exemplo, os pacientes passam no mesmo dia nos vários lugares.

"Porta do PS fechado" - só receber casos que demandem atendimento hospitalar - no mínimo - Triagem de PS

- Incapacidade do poder público gerir a saúde. Incluem-se as opiniões que apontam falta ou má gestão dos recursos da saúde, corrupção, baixos salários dos médicos e necessidade de uma maior autonomia do trabalho médico como causas dessa inadequação.

O não investimento financeiro ou a corrupção que não permitem melhoria nestes investimentos (não chega o recurso porque deve ser desviado). Que nossos políticos se preocupassem realmente com o problema da saúde. Não só no palanque.

Assistência médica ambulatorial, pública e privada, inadequada, causada por: a) investimento público insuficiente. b) Desvio de recursos públicos e de recursos pessoais, a fundo perdido, favorecendo empresas de saúde. c) Aviltamento de exercício profissional médico complementar à saúde pública.

Seria necessária permissão do exercício profissional liberal autônomo e livre concorrência.

Falta dinheiro para o paciente escolher. Vontade e disposição política para uma mudança constitucional (a saúde não é obrigação do Estado). Nova distribuição. Não dar nada. Esclarecimento da população (sem gasto excessivo!)

Dar à saúde a prioridade devida. Verbas e atitude politica, vontade. Dinheiro não falta!!!

\section{B) Profissionais das UBS}

Respostas às perguntas $1 \mathrm{e} 2 \mathrm{e}$ respectivas categorias são descritas a seguir. Nas tabelas 3 e 4, encontram-se as frequências das categorias, segundo profissional e município, relacionadas às perguntas 1 e 2 , respectivamente.

Em relação à pergunta 1 (Considerando a necessidade de melhor delimitar as funções das Unidades Básicas de Saúde e dos Prontos-Socorros, nos casos de febre, pródromos de IVAS, broncoespasmo, sintomas de descontrole de hipertensão ou diabetes, para qual desses dois serviços as pessoas, em geral, deveriam ser orientadas a procurar atendi- 
mento?), as respostas emitidas pelos profissionais foram classificadas em três categorias:

- Procurar a UBS. Foram incluídas as opiniões que consideraram que esse atendimento era atribuição da UBS, ainda que se afirmando a necessidade de organização e acertos para que o mesmo fosse realizado adequadamente, propondo-se continuidade dos cuidados e vinculação às unidades básicas.

Desde que haja disponibilidade de vagas, todas essas patologias podem e devem ser atendidas na UBS.

Se possivel, estas intercorrências deveriam ser atendidas pelo próprio médico que acompanha a criança.

Primeiramente para as unidades básicas; caso fosse uma descompensação intensa seria encaminhado para PS.

Em todos os casos os pacientes poderiam dirigir-se primeiramente às unidades de referência para avaliação da situação e atendimentos possíveis na própria UBS, mesmo sendo necessária remoção. Dessa forma existe mais uma oportunidade para o profissional saber o que ocorre com seus pacientes e planejar o acompanhamento.

A UBS deveria ter organização e pessoal suficiente para atender todos esses casos de uma forma inicial.

- Procurar a UBS e o PS/AMA. Nesta categoria foram incluídos os discursos que, segundo um juízo particular em relação a cada ocorrência, estabeleciam condições ou gradientes relacionados à doença, indicando um ou outro tipo de serviço (UBS ou PS/AMA) a ser procurado pela população.

No caso de febre e pródromos de IVAS as pessoas deveriam encaminhar-se às unidades básicas de saúde. No caso de broncoespasmos, sintomas de descontrole de hipertensão ou diabetes deveriam ser orientadas a procurar atendimento nos prontos-socorros.

Hipertensão, diabetes e IVAS, procurar a unidade; febre e broncoespasmo o pronto-socorro.

Os casos de broncoespasmo devem ser encaminhados ao pronto-socorro. Descontrole de HAS e diabetes deverão ser avaliados pelo clínico da unidade.

febre = UBS ou PS; broncoespasmos =PS; pródromos de IVAS = UBS; sintomas de descontrole de HAS ou diabetes $=$ PS ou UBS.

- Procurar PS/AMA. Nesta categoria incluíram-se as opiniões dos profissionais que explicitavam que não é atribuição da UBS prestar este tipo de atendimento e que reforçavam um papel da UBS limitado às ações de promoção, preven- ção, colocando-as em confronto com o atendimento da demanda não agendada.

Deveriam ser orientadas a procurar o prontosocorro.

A unidade básica de saúde como o próprio nome diz deveria atender com a finalidade de prevenção e acompanhamento ambulatorial de pacientes com patologias crônicas, mas controladas.

Deveriam ser orientados a procurar o PS, pois ali existem serviços que na UBS não tem.

Nestes casos como são urgências - febre, crise hipertensiva, broncoespasmo - deve-se procurar unidades apropriadas para tal: PS, PA.

Para os pronto-atendimentos, nem para as UBS nem para o PS.

Nas UBS só deveriam ser atendidas as consultas marcadas.

Quanto à pergunta 2 (Costuma-se afirmar que grande parte da demanda atendida em PS/AMA são ocorrências ou doenças que deveriam ser atendidas em unidades básicas de saúde. Qual sua opinião sobre isso?), as respostas emitidas pelos profissionais foram classificadas em seis categorias, as quais constituíram dois grupos:

Grupo 1 - Opinam que essa demanda deveria ser atendida na UBS - neste grupo foram incluídos os discursos que afirmaram que essa demanda deveria ser atendida na UBS, segundo três entendimentos distintos categorizados e descritos a seguir.

- Numa primeira categoria encontram-se discursos daqueles que consideram que o problema gerador dessa demanda atendida desnecessariamente nos serviços de urgência e emergência está na incapacidade de a população compreender o papel de cada um desses serviços - unidade básica de saúde e PS/AMA. Decorre isto da falta de orientação das pessoas, sendo fundamental o comparecimento às consultas de rotina na UBS para que elas possam cuidar de sua saúde e reconhecer sinais e sintomas que indiquem o local mais apropriado para o atendimento - UBS ou o PS/AMA. Apontam para a necessidade de campanhas educativas com a utilização de diferentes meios de comunicação.

- Numa segunda categoria encontram-se discursos daqueles que consideram que o problema está na falta de recursos das unidades básicas no que se refere a sua estrutura, equipamentos disponíveis e, sobretudo, profissionais em número e com qualificação adequados; há um desequilíbrio entre a oferta de serviços e a demanda. É necessário equipar as unidades, melhorar as condições de trabalho, contratar e capacitar os profissionais que atuam nas UBS. 
- Numa terceira categoria do Grupo 1 encontram-se discursos daqueles que consideram que o problema está no processo de trabalho. Referem que a UBS não tem uma recepção técnica acolhedora, de funcionamento contínuo, com protocolos estabelecidos, capaz de apreender as questões e problemas da demanda não agendada. Não organiza, também, formas de manter espaços nas agendas médicas para atendimento dessa demanda ou profissionais definidos para o mesmo. Há pouco envolvimento de equipe multiprofissional na assistência e os aspectos administrativos persistem como critérios na definição do atendimento a essa demanda. As agendas tem pouca disponibilidade de vagas e há muitas barreiras que dificultam o agendamento.

Grupo 2 - Opinam que essa demanda NÃO deveria ser atendida na UBS - neste grupo foram incluídos os discursos que afirmaram que essa demanda não deveria ser atendida na UBS, segundo dois entendimentos distintos categorizados e descritos a seguir.

- Numa primeira categoria do Grupo 2 encontram-se os discursos daqueles que consideram que a UBS tem papel de promoção da saúde e prevenção, deve trabalhar com agendamento e não deve realizar consultas para afecções que necessitam de atendimento imediato não agendado. Consideram que se a UBS atuasse adequadamente, com seu funcionamento pleno e a população comparecesse às consultas, seguissem as orientações, essas doenças não ocorreriam. No funcionamento pleno apontam a necessidade de profissionais em número adequado, exames subsidiários e medicamentos. É feita uma crítica à excessiva procura, sem necessidade, de PS/AMA pela população.

- Numa segunda categoria do Grupo 2 encontram-se os discursos daqueles que consideram que essas ocorrências não deveriam ser atendidas em PS/AMA, mas que também não deveriam ser atendidas na UBS, pois consideram que nestes serviços não há condições. Propõem que deva existir um pronto-atendimento, intermediário, entre a UBS e o PS, citando o tipo de unidade AMA como opção para esse atendimento e reforçando que a UBS deve ser reservada para consultas agendadas.

\section{Discussão}

São questões estratégicas para a organização do sistema de saúde brasileiro, já amplamente reconhecidas, o papel, a extensão e a organização que a rede básica deve assumir ${ }^{15}$. Desenvolver uma organização de serviços e uma prática comprometida com a integralidade do cuidado é um problema central ${ }^{1,16}$. A concretização do princípio da integralidade requer a superação do entendimento da rede básica como sinônimo de equipe mínima, simplificada, com baixa resolubilidade, sem trabalho em equipe multiprofissional. Um discurso exacerbado da atenção básica limitada à promoção de saúde em contraposição às ações assistenciais e que se recusa a atender de forma sistemática à demanda não agendada resulta na incapacidade em abrir acesso e acolher as pessoas, assumindo a responsabilidade pela saúde da população de uma área e oferecendo cuidados integrais e contínuos.

A diversidade de locais de trabalho, a quantidade dos participantes deste estudo (296 profissionais) e o encadeamento das questões indagadas nas entrevistas possibilitaram um entendimento amplo das diferentes concepções dos profissionais de saúde. Tal condução metodológica qualitativa dispensa a necessidade de amostras mais numerosas ou probabilísticas para a compreensão do fenômeno em questão, isto é, as visões dos profissionais e como elas podem influenciar os comportamentos e a definição da sistemática do processo de trabalho nos serviços de atenção básica.

Os 106 médicos atuantes em unidade de urgência-emergência entrevistados, após indicarem um conjunto de afecções costumeiramente atendidas nessas unidades, mas que deveriam ser atendidas nas UBS, opinaram, em apenas $14,5 \%$ das vezes, que essa situação seria uma decorrência da falta de informação da população. O juízo dessa situação, por esses profissionais, concentra-se em problemas de insuficiência e inadequação de recursos na UBS. Essa maneira de pensar o problema é muito similar entre os médicos nas unidades de urgência-emergência dos três municípios pesquisados, o que parece oferecer robustez a esse tipo de achado (Tabela 2).

Quando os médicos, os enfermeiros e o pessoal de enfermagem de UBS são questionados sobre o papel dessas unidades em atender essas afecções indicadas pelos médicos de unidades de urgência-emergência (Quadro 1, sintetizado nas situações de febre, pródromos de IVAS, broncoespasmo, sintomas de descontrole de hipertensão ou diabetes) foi possível organizar os discursos de modo a compreender um conflito básico entre eles, anterior à discussão do porquê isso ocorreria. Não há concordância sobre o fato de onde, preferencialmente, essas situações clássicas de 
demanda não agendada deveriam ser atendidas. $\mathrm{Na}$ Tabela 3, pode-se observar que apenas $1 / 3$ dos entrevistados são favoráveis ao atendimento dessas situações na UBS e que é, especialmente, diferente a visão entre os profissionais médicos e os enfermeiros.

Entretanto, essa não indicação de que essas situações deveriam ser orientadas a buscar atendimento nas UBS poderia ser resultante não da concepção que os entrevistados tem sobre o papel dessas unidades no sistema de saúde, mas como decorrência da situação concreta de possíveis deficiências vividas nessas unidades, tal como majoritariamente apontado pelos médicos socorristas. Assim, para os profissionais de UBS esse acréscimo de demanda não se justificaria dada a escassez dos recursos existentes. Por esta razão é particularmente esclarecedora a questão indagada na sequência. A fim de apurar qual era a concepção dos profissionais de UBS entrevistados, perguntou-se a eles sobre o que pensa- vam e o porquê da afirmação corrente de que grande parte da demanda atendida em PS/AMA são ocorrências ou doenças que deveriam ser atendidas em unidades básicas de saúde.

Com tal indagação foi possível categorizar as respostas de modo a deixar mais evidente e clara a diferença de opiniões. Após concordarem ou não com a afirmação, discursaram sobre o porquê e, assim, foi possível identificar aqueles que não concordavam que essa demanda deveria ser atendida nas UBS, por considerá-la conceitualmente fora de suas atribuições e não apenas por razões conjunturais de insuficiência de recursos nas UBS. Na Tabela 4 observa-se que após esse procedimento, ainda assim, 44\% dos entrevistados consideraram que essa demanda não cabe à UBS.

Esse discurso, muito frequente entre os profissionais das unidades básicas, é em parte justificado afirmando-se que tal demanda não agendada na UBS poderia ser equacionada se hou-

Tabela 3. Discursos dos profissionais de UBS, segundo indicação do serviço de saúde a ser utilizado em determinadas afecções*. Projeto MABS - Região Metropolitana de São Paulo, 2006-2008.

\begin{tabular}{|c|c|c|c|c|c|c|c|c|}
\hline \multirow[b]{2}{*}{ Indicação } & \multicolumn{2}{|c|}{ Enfermagem ${ }^{* *}$} & \multicolumn{2}{|c|}{ Enfermeiro } & \multicolumn{2}{|c|}{ Médico } & \multicolumn{2}{|c|}{ Total } \\
\hline & $\mathbf{N}$ & $\%$ & $\mathbf{N}$ & $\%$ & $\mathbf{N}$ & $\%$ & $\mathbf{N}$ & $\%$ \\
\hline $\mathrm{Na}$ UBS & 15 & 34,1 & 11 & 68,8 & 36 & 28,6 & 62 & 33,3 \\
\hline Na UBS e no PS, AMA & 9 & 20,5 & 3 & 18,8 & 25 & 19,8 & 37 & 19,9 \\
\hline No PS, AMA & 20 & 45,5 & 2 & 12,5 & 65 & 51,6 & 87 & 46,8 \\
\hline Total & 44 & 100,0 & 16 & 100,0 & 126 & 100,0 & 186 & 100,0 \\
\hline
\end{tabular}

Ignorado $=4$

“Afecções indagadas: casos de febre, pródromos de IVAS, broncoespasmo, sintomas de descontrole de hipertensão ou diabetes.

${ }^{* *}$ Enfermagem = auxiliar e técnico de enfermagem

Tabela 4. Discursos dos profissionais de UBS, segundo categoria temática explicativa da demanda nos PS e AMAS por afecções características do papel da atenção básica. Projeto MABS - Região Metropolitana de São Paulo, 2006-2008.

\begin{tabular}{|c|c|c|c|c|c|c|c|c|}
\hline \multirow[b]{2}{*}{ Categoria temática } & \multicolumn{2}{|c|}{ Enfermagem ${ }^{*}$} & \multicolumn{2}{|c|}{ Enfermeiro } & \multicolumn{2}{|c|}{ Médico } & \multicolumn{2}{|c|}{ Total } \\
\hline & $\mathbf{N}$ & $\%$ & $\mathbf{N}$ & $\%$ & $\mathbf{N}$ & $\%$ & $\mathbf{N}$ & $\%$ \\
\hline Grupo 1 - É Papel da UBS. Há essa demanda por: & 25 & 58,1 & 13 & 81,3 & 64 & 52,0 & 102 & 56,0 \\
\hline Desconhecimento da população no uso dos serviços & 3 & 7,0 & 1 & 6,3 & 10 & 8,1 & 14 & 7,7 \\
\hline Insuficiência de recursos nas UBS & 16 & 37,2 & 3 & 18,8 & 35 & 28,5 & 54 & 29,7 \\
\hline Inadequação do processo de trabalho das UBS & 6 & 14,0 & 9 & 56,3 & 19 & 15,4 & 34 & 18,7 \\
\hline Grupo 2 - Não é papel da UBS. Há essa demanda por: & 18 & 41,9 & 3 & 18,8 & 59 & 48,0 & 80 & 44,0 \\
\hline Falta de reforço no papel de promoção da saúde nas UBS & 17 & 39,5 & 3 & 18,8 & 35 & 28,5 & 55 & 30,2 \\
\hline Falta de unidades intermediárias (PA) entre UBS e PS & 1 & 2,3 & 0 & - & 24 & 19,5 & 25 & 13,7 \\
\hline Total & 43 & 100,0 & 16 & 100,0 & 123 & 100,0 & 182 & 100,0 \\
\hline
\end{tabular}

Ignorado $=8$

* Enfermagem $=$ auxiliar e técnico de enfermagem 
vesse incentivo à promoção da saúde e à prevenção. Tal entendimento aposta que a promoção e a prevenção resolveriam a questão num futuro, entretanto para o problema no presente descartam que caberia à UBS atender essa demanda existente.

Em decorrência dessas diversas concepções, fica evidente que as orientações obtidas pelos usuários nos serviços básicos de saúde, diante de diferentes situações, serão confusas e contraditórias, predominando uma orientação restritiva à utilização das unidades básicas, situação esta, também, apontada em outros estudos ${ }^{17}$.

Alguns profissionais consideraram que deveria ocorrer uma mudança no processo de trabalho das UBS, que incluísse acolhimento, atendimento do não agendado, trabalho em equipe multiprofissional, definição de protocolos. Outros, entretanto, defenderam que a solução estaria em propostas de educação da população para que ela aprendesse a utilização adequada dos serviços de saúde e não se valesse da consulta não agendada na UBS.

Muitos dos que afirmaram que não era papel da UBS assumir tal demanda enfatizaram que isto poderia ser adequadamente resolvido com a ampliação de unidades de pronto-atendimento que, de fato, vieram a ganhar forte prioridade de recursos na região. Nesse contexto, deve-se enfatizar a importância do endosso que gestores vêm propiciando a esse entendimento com a valorização e a expansão de unidades de pronto-atendimento, intermediárias entre as UBS e as PS e caracterizadas por realizar atendimento pontual, segundo o modelo queixa-conduta.

A demanda excessiva, e considerada não adequada aos serviços de urgência e emergência, tem sido constatada para todas as faixas etárias e ocorre no Brasil, em outros países em desenvolvimento e, também, nos países desenvolvidos ${ }^{18-22}$. Os percentuais de não adequação variam entre os estudos a depender do critério empregado. Essa variação se deve sobretudo às controvérsias quanto aos níveis assistenciais a serem utilizados nas diferentes situações, tanto da parte dos usuários, como dos profissionais, conforme verificado também em nosso estudo.

\section{Conclusões}

Os entrevistados, exercendo atividades em diferentes tipos de serviço, expressaram opiniões contraditórias sobre o papel da atenção básica em relação ao atendimento da demanda não agendada. Esse conflito foi mais intenso entre os médicos, em menor grau no pessoal de enfermagem e em grau menor ainda entre os enfermeiros que, dentre os entrevistados, apareceram como o grupo mais homogêneo, em termos de visão do papel da UBS.

Os discursos evidenciaram elementos importantes para a compreensão das visões coexistentes sobre os serviços básicos. Essas visões disputam, segundo variados interesses, o significado a ser atribuído, na prática, à abrangência da atenção básica. A maior força de uma ou de outra visão está ligada ao processo de condução das mudanças para a efetivação integral do direito à saúde e a organização de uma rede de atenção efetiva com claras atribuições aos seus pontos de atenção.

A abrangência das funções da atenção básica é um signo em disputa e a recusa ao atendimento da demanda não agendada reflete posicionamentos que compõem o complexo de determinação de possibilidades ou restrições na reorientação do trabalho nas unidades básicas.

Refletindo nessas direções, fica evidente que as visões identificadas integram-se ao conjunto de questões que permeiam a ampliação do acesso na atenção básica. Esse conjunto não se reduz ao conhecimento de recursos disponíveis, aos processos normalizados burocraticamente ou ao volume de produção de atos e procedimentos, aspectos necessários, mas não suficientes para a compreensão da estruturação da atenção básica em cada local. É impossível compreender os problemas da organização sem considerar a visão dos sujeitos envolvidos e as relações que se estabelecem entre as pessoas e seus grupos de interesse como parte da totalidade do funcionamento dessa política social.

A resposta imediata que a população procura nos serviços básicos é uma mudança que não é simples nem consensual, envolve interesses diversos, exige canais de participação crítica e decisão entre gerentes, equipe de saúde e cidadãos/ usuários. 


\section{Colaboradores}

PT Puccini, VK Cornetta, TZ Sahyom, ICP Fuentes, LMG Botta, RF Puccini participaram do planejamento, acompanhamento da coleta de dados e discussão dos resultados. PT Puccini trabalhou na concepção e metodologia do estudo. PT Puccini e RF Puccini foram responsáveis pela análise dos dados e redação final do artigo.

\section{Referências}

1. Puccini PT, Cecílio LCO. A humanização dos serviços e o direito à saúde. Cad Saude Publica 2004; 20(5):1342-1353.

2. Gomes MCPA, Pinheiro R. Acolhimento e vínculo: práticas de integralidade na gestão do cuidado em saúde em grandes centros urbanos. Interface Comunic Saúde Educ 2005; 9(17):287-301.

3. Conill EM. Complementando a discussão sobre a atenção básica: pode o acesso, a integralidade e o controle social se constituírem em temáticas de consenso para a avaliação da reforma brasileira? Cien Saude Colet 2002; 7(3):421-423.

4. Souza ECF, Vilar RLA, Rocha NSP, Uchoa AC, Rocha PM. Acesso e acolhimento na atenção básica: uma análise da percepção dos usuários e profissionais de saúde. Cad Saude Publica 2008; 24(Supl. 1):S100-S110.

5. Furtado BMASM, Araújo Jr JLC, Cavalcanti P. O perfil da emergência do Hospital da Restauração: uma análise dos possíveis impactos após a municipalização dos serviços de saúde. Rev Bras Epidemiol 2004; 7(3):279-289.

6. Pires MRGM, Göttems LBD, Martins CMF, Guilhem D, Alves ED. Oferta e demanda por média complexidade/SUS: relação com atenção básica. Cien Saude Colet 2010; 15(Supl. 1):1009-1019.

7. Gérvas J. Cuidados primários de saúde na Europa: tendências atuais. In: Seminário Internacional sobre Cuidados Básicos de Saúde - 25 anos de Declaração de Alma-Ata. Brasília (DF), 2003. Rev Bras Epidemiol 2004; 7(3):350-369.

8. Gonçalves RBM. Tecnologia e organização social das práticas de saúde. São Paulo: Editora Hucitec, Abrasco; 1994.

9. Takemoto MLS, Silva EM. Acolhimento e transformações no processo de trabalho de enfermagem em unidades de saúde de Campinas. Cad Saude Publica 2007; 23(2):331-340.

10. Camelo SHH, Angerami ELS, Silva EM, Mishima SM. Acolhimento à clientela: estudo em unidades básicas de saúde no município de Ribeirão Preto. Rev Latino-Am Enfermagem 2000; 8(4):30-37.

11. Puccini PT. As unidades de assistência médica ambulatorial (AMA) do Município de São Paulo, Brasil: condições de funcionamento e repercussões sobre a atenção básica no Sistema Único de Saúde, 2006. Cad Saude Publica 2008; 24(12):2755-2766.

12. Pucini PT, Cornetta VK. Ocorrências em prontosocorro: eventos sentinela para o monitoramento da atenção básica de saúde. Cad Saude Publica 2008; 24(9):2032-2042.

13. Fundação SEADE. Informações sobre Municípios Paulistas. 2012. [site na Internet]. [acessado 2012 mar 20]. Disponível em: http://www.seade.gov.br/ produtos/imp/

14. Bardin L. Análise de conteúdo. Lisboa: Edições 70; 2004.

15. Bodstein R. Atenção básica na agenda da saúde. Cien Saude Colet 2002; 7(3):401-412.

16. Hartz ZMA, Contandriopoulos AP. Integralidade da atenção e integração de serviços de saúde: desafios para avaliar a implantação de um "sistema sem muros”. Cad Saude Publica 2004; 20(Supl. 2):S331-S336. 
17. Oliveira LH, Mattos RA, Souza AIS. Cidadãos peregrinos: os "usuários" do SUS e os significados de sua demanda a prontos-socorros e hospitais no contexto de um processo de reorientação do modelo assistencial. Cien Saude Colet 2009; 14(5):1929-1938.

18. Vera CF, Werner AF, Massri DE. Complejidad de las consultas en un servicio de urgencia con consultorio municipal adosado. Cuad Cir 2004; 18(1):27-32.

19. Burnet MG, Grover AS. Use of the emergency department for no urgent care during regular business hours. CMAJ 1996; 154(9):1345-1351.

20. Gill JM, Riley AW. No urgent use of hospital emergency departments: urgency from patient's perspective. J Fam Pract 1996; 42(5):491-495.

21. Simons DA, Monlleo IL, Simons SA, Araújo Junior JL. Adequação da demanda de crianças e adolescentes atendidos na Unidade de Emergência em Maceió (AL). Rev Bras Saúde Matern Infant 2010; 10(1):59-67.

22. Souza BC, Felippe EBA, Silva RM. Perfil da demanda do Departamento de Emergência do Hospital Nossa Senhora da Conceição - Tubarão (SC). ACM arq catarin med 2009; 38(2):73-79.

Artigo apresentado em 30/04/2012

Aprovado em 17/07/1012

Versão final apresentada em 30/08/2012 